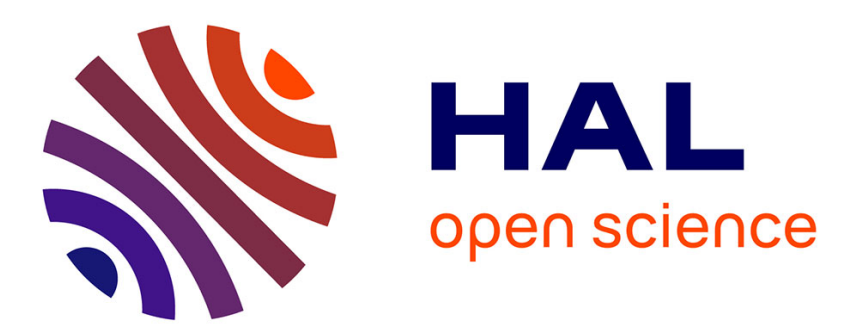

\title{
An adaptive observer for a class of nonlinear systems with a high-gain approach. Application to the twin-rotor system
}

Habib Dimassi, Salim Hadj Saï, Antonio Loria, Faouzi M'Sahli

\section{- To cite this version:}

Habib Dimassi, Salim Hadj Saïd, Antonio Loria, Faouzi M'Sahli. An adaptive observer for a class of nonlinear systems with a high-gain approach. Application to the twin-rotor system. International Journal of Control, 2021, 94 (2), pp.370-381. 10.1080/00207179.2019.1594387 . hal-02367534

\section{HAL Id: hal-02367534 \\ https://hal.science/hal-02367534}

Submitted on 5 Mar 2020

HAL is a multi-disciplinary open access archive for the deposit and dissemination of scientific research documents, whether they are published or not. The documents may come from teaching and research institutions in France or abroad, or from public or private research centers.
L'archive ouverte pluridisciplinaire HAL, est destinée au dépôt et à la diffusion de documents scientifiques de niveau recherche, publiés ou non, émanant des établissements d'enseignement et de recherche français ou étrangers, des laboratoires publics ou privés. 


\title{
An adaptive observer for a class of nonlinear systems with a high gain approach. Application to the twin-rotor system
}

\author{
Habib Dimassi, ${ }^{a, b}$ Salim Hadj Said, ${ }^{a}$ Antonio Loría ${ }^{c}$ and Faouzi M'Sahli. ${ }^{a}$ \\ a University of Monastir, Ecole Nationale d'Inénieurs de Monastir, ESIER, 5019, Monastir, Tunisia. \\ ${ }^{\mathrm{b}}$ University of Sousse, Institut supérieur des sciences appliquées et de technologie de Sousse. Tunisia. \\ ${ }^{c}$ CNRS, LSS-Supelec, 3 Rue Joliot-Curie, 91192 Gif sur Yvette, France.
}

\author{
ARTICLE HISTORY \\ Compiled October 7, 2018
}

\begin{abstract}
In this paper, we investigate the problem of adaptive observer design for a class of nonlinear systems subject to unknown parameters and such that the classical observer matching assumption is not satisfied. That is, it is assumed that the relative degree of the outputs with respect to the unknown parameters vector is at least equal to two. We adopt the idea of generating auxiliary outputs based on a high gain observer. The generated outputs are employed by a new adaptive observer to reconstruct both the states and unknown parameters. The stability analysis of the system error is established based on a Lyapunov analysis. It is shown that the state estimation error and the adaptation error are uniformly bounded and converge to a compact set that may be reduced by an appropriate choice of the design parameters. In order to improve the robustness of our approach, the proposed adaptive observer is appropriately modified based on sliding modes theory to compensate for the effect of the time-varying and bounded disturbances. Theoretical results are illustrated and validated for the twin rotor MIMO system with numerical simulations.
\end{abstract}

\section{KEYWORDS}

Adaptive observer, high gain observers, observer matching condition, unknown parameters, twin rotor MIMO system.

\section{Introduction}

The problem of adaptive observer design for nonlinear systems is one of the challenging problems in the literature of automatic control and has many important applications such that identification, fault detection, fault tolerant control and chaos-based communications. Different approaches have been developed in the literature of automatic control. In [1], an adaptive observer have been proposed for a class of MIMO linear time-varying systems under the persistency of excitation condition; this approach doesn't need any structural condition but it is only valid for linear time-varying systems. On the other hand, adaptive high gain observers nonlinear systems with linear parameterization have been presented in [2] and [3] for a particular class of nonlinear systems in the triangular canonical form. Then, the authors of [4] have proposed adaptive observers for a class of uniformly observable nonlinear MIMO systems with linear and/or nonlinear parameterization. Other contributions on the design of 
adaptive high gain observers with nonlinear prametrizations have been also developed in [5] and [6]. In more recent works, different extensions of adaptive high gain observers have been designed in the case of uniformly observable systems with sampled outputs in [7] and for the case of non uniformly observable nonlinear systems based on the augmented model approach in [8]. In another research work, an adaptive observer scheme was applied to a class of systems (including chaotic systems) such that the dynamics contains three terms [9]: the first term depends linearly in the unmeasured states, the second depends nonlinearly in the unmeasured states and linearly in the unknown parameters and a third term is considered to depend both on time and (un)measured states without parametric uncertainty. The latter approach was applied to the synchronization of chaotic systems under the persistency of excitation assumption by exploiting the richness of chaotic systems. More recently, a new adaptive estimation approach was presented in [10] for a class of nonlinear systems where nonlinear and regressor terms depend only on the output signal, then based on sliding modes techniques, the approach was extended to the case where the system is subject to unknown disturbances.

An important class of adaptive observers have been addressed to a more general class of systems satisfying the Lipschitz condition and the persistency of excitation assumption (See [11] and [12]). Such adaptive observers are designed under the necessary and sufficient conditions that the invariant zeros of the system are in the open left half complex plane and that the relative degree of the outputs with respect to the unknown parameters vector is equal to one. The relative degree condition is a restrictive assumption that limits the applicability of the latter class of adaptive observers. Indeed, in many physical systems and particularly in mechanical systems, the observer matching condition is not verified. However, the advantage of this approach is its simpler architecture for implementation compared to all other adaptive estimation approaches.

In this paper, we consider the problem of adaptive observer design for a class of nonlinear systems subject to unknown parameters such that the observer matching condition is not satisfied. Our main contribution consists in relaxing the latter restrictive assumption and to propose a new adaptive estimation approach for joint states and unknown parameters estimation for nonlinear systems with relative degree $\geq 2$. For this purpose, we adopt the idea of generating auxiliary outputs and their estimates based on a high gain observer in order to make the relative degree assumption satisfied. This idea was first proposed by the authors of [13] to solve the problem of first order sliding mode observer design for linear systems subject to unknown inputs and such that the observer matching condition is not verified. In this paper, the estimated auxiliary outputs generated by the latter high gain observer are used by the main adaptive observer proposed in this paper for which we associate an appropriate adaptation which is designed based on a $\sigma$-modification technique, in order to reconstruct both the states and the unknown parameters. We show that the state estimation and the unknown parameters adaptation errors of the proposed high-gain observer based adaptive observer are uniformly bounded with respect to a compact set which may be reduced for an appropriate choice of the design parameters. The second contribution of this paper consists on improving the robustness of the proposed adaptive estimation approach by investigating the case where the considered system is corrupted by unknown time-varying and bounded disturbances. Inspired from sliding modes theory, we inject a discontinuous input in the dynamics of the adaptive observer in order to compensate for the effect of the unknown disturbances. The modified high-gain approximate differentiator based adaptive observer allows to reconstruct both the states and the unknown parameters despite the presence of the unknown disturbances.

The remainder of this paper is organized as follows. In section 2, we formulate the problem 
statement. In section 3, we introduce our main results consisting on our proposed adaptive observer, the stability analysis as well as the improvement of robustness against perturbationsss. In section 4, we apply and we validate our theoretical results for the twin rotor MIMO system with numerical simulations. Finally, we conclude with some remarks in section 5 .

Notation. We use the following notations throughout the paper. $|\cdot|$ denotes the absolute value for scalars, the induced norm for matrices and the euclidean norm for vectors.

$I_{n}$ and $0_{n}$ represent respectively the identity and the zero matrices of size equal to $n$.

$\lambda_{\min }(X)$ and $\lambda_{\max }(X)$ denote the minimal and the maximal eigenvalues of a matrix $X$.

\section{Context and problem formulation}

\subsection{Background on adaptive observers for a class of nonlinear systems}

Consider the following class of uncertain nonlinear systems,

$$
\left\{\begin{array}{l}
\dot{x}=A x+B f_{0}(x, u)+B g_{0}(x, u) \theta+E u \\
y=C x
\end{array}\right.
$$

where $x \in \mathbb{R}^{n}, y \in \mathbb{R}^{p}, u \in \mathbb{R}^{r}, \theta \in \mathbb{R}^{q}$ are respectively the state, the measured output, the unmeasured state, the known input vector and the unknown constant parameters vector. $A, B$, $C$ and $E$ are constant matrices of appropriate dimensions ( $B, C$ and $D$ are assumed to be of full rank). Let us now consider the following classical assumptions usually considered in the literature of adaptive and unknown inputs observers.

Assumption 1. The nonlinear functions $f_{0}$ and $g_{0}$ satisfy the Lipschitz condition in $x$, uniformly with respect to $u$ with Lipschitz constants $K_{f}$ and $K_{g}$ respectively. That is, $\forall x, \hat{x} \in \mathbb{R}^{n}$,

$$
\begin{aligned}
& \left|f_{0}(x, u)-f_{0}(\hat{x}, u)\right| \leq K_{f}|x-\hat{x}| \\
& \left|g_{0}(x, u)-g_{0}(\hat{x}, u)\right| \leq K_{g}|x-\hat{x}|
\end{aligned}
$$

Assumption 2. The unknown parameters vector $\theta$ is bounded such that for all $t \geq 0$

$$
|\theta(t)| \leq K_{\theta}
$$

Furthermore, it is assumed that $\theta(t)$ is a slowly varying function, that is $\dot{\theta}(t) \simeq 0$

Assumption 3. The function $B g_{0}(\hat{x}(t), u(t))$ is persistently exciting. That is, there exist $\mu>0$ and $T>0$ such that

$$
\int_{t}^{t+T} g_{0}(\hat{x}(s), u(s))^{\top} B^{\top} B g_{0}(\hat{x}(s), u(s)) d s \geq \mu \quad \forall t \geq 0 .
$$

Assumption 4. The invariant zeros of the system model given by the triple $(A, B, C)$ are in the open left-hand complex plane. That is,

$$
\operatorname{Rank}\left[\begin{array}{cc}
A-\lambda I & B \\
C & 0
\end{array}\right]=n+\operatorname{Rank}[B]
$$

for each complex number $\lambda$ such that $\operatorname{Re}(\lambda) \geq 0$ 
Assumption 5. The observer matching condition is satisfied. That is,

$$
\operatorname{Rank}[C B]=\operatorname{Rank}[B]
$$

Under Assumptions 1-5, and based on the design procedure from [11] and [12], one can construct the following adaptive observer for the system (1):

$$
\dot{\hat{x}}=A \hat{x}+B f_{0}(\hat{x}, u)+B g_{0}(\hat{x}, u) \hat{\theta}+L(y-C \hat{x})
$$

and the adaptation law

$$
\dot{\hat{\theta}}=\delta g_{0}(\hat{x}, u)^{T} M(y-C \hat{x})
$$

The constant $\delta$ is a positive real number imposed by the designer. The design matrices $M$ and $L$ are chosen such that

$$
\begin{gathered}
(A-L C)^{T} P+P(A-L C)=-2 Q \\
B^{T} P=M C \\
|B|\left(K_{f}+K_{g} K_{\theta}\right)<\frac{\lambda_{\min }(Q)}{\lambda_{\max }(P)}
\end{gathered}
$$

for some symmetric positive definite matrices $P$ and $Q$.

Assumptions 1-5 are commonly used for the design of the class of adaptive observers (6) and clearly restrict their range of application (1) . Assumption (1) is the well-known Lipschitz condition. Assumption 3 is the classical strong hypothesis of persistency of excitation usually employed in the literature of adaptive control, which means that the regressor function $g_{0}(x)$ should be sufficiently rich in frequencies in order to ensure asymptotic stability and parameter convergence. Assumptions 4 and 5 are structural restrictive conditions meaning that the triple $(A, B, C)$ is minimum phase and relative degree one. In this paper, we focus on the problem of designing adaptive observers based on the form (6) under more relaxed assumptions. Indeed, as we will show in the next subsection, Assumption (1) may be replaced by more relaxed assumptions under which we can apply the so-called Lipscitz extension. On the other hand, a modified alternative structure of the adaptation law (7) based on a $\sigma$-modification technique will be employed in order to avoid the restrictive persistency of excitation assumption 3. Finally, in our adaptive estimation approach that we propose in this paper, the structural strong assumption 5 will be weakened based on the idea of generating auxiliary output and on the design of a high gain approximate differentiator to estimate them. In the following subsection, we state the problem considered in this paper and we introduce a motivating example: MIMO the twin rotor system to highlight our main contributions.

\subsection{Problem statement}

In this paper, we consider the class of nonlinear systems (1). In order to extend the range of applications of our adaptive estimation approach that we propose later, we make the following more relaxed assumptions instead of Assumption 1:

Assumption 6. The nonlinear functions $f_{0}$ and $g_{0}$ are assumed to be once continuously differentiable with respect to their arguments. 
Assumption 7. The solutions to the system (1) as well as the input function $u$ are assumed to be uniformly bounded.

We use the assumptions 6 and 7 to apply the so-called Lipschitz-extension technique for the nonlinear functions $f_{0}$ and $g_{0}$ (See for instance [4], [14], [15]). To that end, let $\eta: \mathbb{R}^{n} \rightarrow X$ be a linear saturation such that $\eta(x)=x$ for all $x \in X$ where $X=\left\{x \in \mathbb{R}^{n},\left|x_{i}\right| \leq d_{i}, i=1, \cdots, n\right\}$ with $x_{i}$ is the $\mathrm{i}^{\text {th }}$ component of $x$ and $d_{i}$ are positive constants. Next, we define, respectively, the prolongations $f$ and $g$ of the nonlinear functions $f_{0}$ and $g_{0}$ such that $f(x, u)=f_{0}(\eta(x), u, t)$ and $g(x, u)=g_{0}(\eta(x), u, t)$ for all $x \in X$. Now, under Assumption 7, since $f$ and $g$ are once continuously differentiable and using the arguments of the proof in the appendix of [15], it can be proved based on the mean value theorem that the extended nonlinear functions $f$ and $g$ are globally Lipschitz with respective Lipschitz constants $K_{f}$ and $K_{g}$, i.e $\forall x, \hat{x} \in \mathbb{R}^{n}$, we have

$$
\begin{array}{r}
|f(x, u)-f(\hat{x}, u)| \leq K_{f}|x-\hat{x}| \\
|g(x, u)-g(\hat{x}, u)| \leq K_{g}|x-\hat{x}|
\end{array}
$$

We consider now the following dynamical system

$$
\left\{\begin{array}{l}
\dot{x}=A x+B f(x, u, t)+B g(x, u) \theta+E u \\
y=C x
\end{array}\right.
$$

It is clear that for all $t$ such that $x(t) \in X$, the trajectories of the system (1) coincide with those of the system (13).

In this research work, we focus on the observer matching assumption 5 which means that the relative degree of the outputs with respect to the unknown parameters vector is equal to one. This assumption clearly restricts the applicability of the class of adaptive observers presented in Section 2.1 for many physical systems. In particular, this assumption is clearly not verified for mechanical systems such that self-balancing robots, inverted pendulums, flexible robots, quadrotors and twin rotor system where only positions and angles are measured whereas parameter uncertainties appear in the dynamics of the velocities. Our objective is to solve the problem of joint states and unknown parameters estimation despite that the observer matching condition 5 is not satisfied for System (1).

\subsubsection{Motivating example: the twin rotor MIMO system}

In order to highlight the main contribution of this paper, we consider the nonlinear system consisting on a twin rotor MIMO system described by the following state model:

$$
\left\{\begin{aligned}
\dot{x}_{1}= & x_{2} \\
\dot{x}_{2}= & \frac{a_{1}}{I_{1}} x_{5}^{2}+\frac{b_{1}}{I_{1}} x_{5}-\frac{M_{g}}{I_{1}} \sin \left(x_{1}\right)-\frac{B_{1 \alpha_{v}}}{I_{1}} x_{2} \\
& +\frac{0.0326}{2 I_{1}} \sin \left(2 x_{2}\right) x_{4}{ }^{2}-\frac{k_{g y}}{I_{1}} a_{1} \cos \left(x_{1}\right) x_{4} x_{5}{ }^{2} \\
& -\frac{k_{g y}}{I_{1}} b_{1} \cos \left(x_{1}\right) x_{4} x_{5} \\
\dot{x}_{3}= & x_{4} \\
\dot{x}_{4}= & \frac{a_{2}}{I_{2}} x_{6}{ }^{2}+\frac{b_{2}}{I_{2}} x_{6}-\frac{B_{1 \alpha_{h}}}{I_{2}} x_{4}-\frac{k_{c} a_{1}}{I_{2}} 1.75 x_{5}{ }^{2}-\frac{1.75}{I_{2}} k_{c} b_{1} x_{5} \\
\dot{x}_{5}= & -\frac{T_{10}}{T_{11}} x_{5}+\frac{k_{1}}{T_{11}} u_{v} \\
\dot{x}_{6}= & -\frac{T_{20}}{T_{21}} x_{5}+\frac{k_{2}}{T_{21}} u_{h}
\end{aligned}\right.
$$

where $x_{1}=\alpha_{v}$ is the pitch angle, $x_{2}=\dot{\alpha}_{v}=\Omega_{v}$ is the pitch angular velocity, $x_{3}=\alpha_{h}$ is 
the yaw angle, $x_{4}=\dot{\alpha}_{h}=\Omega_{h}$ is the yaw angular velocity in the horizontal plane, $x_{5}=\tau_{1}$ is the momentum of the main motor, $x_{6}=\tau_{2}$ is the momentum of the tail motor. $u_{1}=u_{v}$ and $u_{2}=u_{h}$ are the control inputs. The numerical values of the physical parameters $a_{1}$, $b_{1}, I_{1}, k_{g y}, a_{2}, b_{2}, I_{2}, B_{1 \alpha_{v}}, B_{1 \alpha_{h}}, k_{c}, k_{1}, k_{2}, T_{10}, T_{11}, T_{20}$ and $T_{21}$ will be given later in Section 4.

We assume that the momentum parameter $M_{g}$ is unknown. The measured outputs are the pitch and the yaw angles $\alpha_{v}$ and $\alpha_{h}$. The state model of the twin rotor MIMO system (14) may be written in the form of (1) with $x=\left[x_{1}, x_{2}, x_{3}, x_{4}, x_{5}, x_{6}\right]^{T}, y=\left[x_{1}, x_{3}\right]^{T}, \theta=M_{g}, u=\left[u_{1}, u_{2}\right]^{T}$, $g_{0}(x, u, t)=\left[g_{01}(x, u, t), 0\right]^{T}, f_{0}(x, u, t)=\left[f_{01}(x, u, t), f_{02}(x, u, t)\right]^{T}$ where

$$
\begin{aligned}
f_{01}(x, u, t)= & \frac{a_{1}}{I_{1}} x_{5}{ }^{2}+\frac{0.0326}{2 I_{1}} \sin \left(2 x_{2}\right) x_{4}{ }^{2}, \\
& -\frac{k_{g} y}{I_{1}} a_{1} \cos \left(x_{1}\right) x_{4} x_{5}{ }^{2}-\frac{k_{g y}}{I_{1}} b_{1} \cos \left(x_{1}\right) x_{4} x_{5} \\
f_{02}(x, u, t)= & \frac{a_{2}}{I_{2}} x_{6}{ }^{2}-\frac{k_{c} a_{1}}{I_{2}} 1.75 x_{5}{ }^{2}, \\
& g_{01}(x, u, t)=-\frac{1}{I_{1}} \sin \left(x_{1}\right),
\end{aligned}
$$

$$
\begin{gathered}
A=\left[\begin{array}{cccccc}
0 & 1 & 0 & 0 & 0 & 0 \\
0 & -\frac{B_{1 \alpha \nu}}{I_{1}} & 0 & 0 & \frac{b 1}{I 1} & 0 \\
0 & 0 & 0 & 1 & 0 & 0 \\
0 & 0 & 0 & -\frac{B_{1 \alpha h}}{I_{2}} & -\frac{1.75}{I_{2}} k_{c} b_{1} & \frac{b_{2}}{I_{2}} \\
0 & 0 & 0 & 0 & -\frac{T_{10}}{T_{11}} & 0 \\
0 & 0 & 0 & 0 & 0 & -\frac{T_{20}}{T_{21}}
\end{array}\right], \\
B=\left[\begin{array}{cccccc}
0 & 1 & 0 & 0 & 0 & 0 \\
0 & 0 & 0 & 1 & 0 & 0
\end{array}\right]^{T} \\
C=\left[\begin{array}{cccccc}
1 & 0 & 0 & 0 & 0 & 0 \\
0 & 0 & 1 & 0 & 0 & 0
\end{array}\right]
\end{gathered}
$$

and

$$
D=\left[\begin{array}{llllll}
0 & 0 & 0 & 0 & 1 & 0 \\
0 & 0 & 0 & 0 & 0 & 1
\end{array}\right]^{T}
$$

It is clear that the observer matching assumption 5 is not satisfied for System (14) since $\operatorname{Rank}(C B) \neq \operatorname{Rank}(B)$ and the relative degree of the output $y$ with respect to the unknown parameters vector $\theta$ is equal to 2 . As a consequence, the adaptive observers of [11] and [12] based on the design procedure presented above in Section 2.1 fail to solve the problem of joint states and unknown parameters since the system of linear matrix equations (26) and (27) is not solvable in this case. Our main objective in this paper is to propose a solution 
to overcome the latter restriction. We will prove later that the problem of joint of states and unknown parameter estimation is successfully solved for the twin rotor system based on our proposed estimation approach despite that Assumption 5 is not satisfied.

\subsubsection{Relaxation of the observer matching condition}

The observer matching assumption 5 is also needed to synthesize classical first order sliding mode observers such as Walcott-Zak sliding mode observer [16] for systems subject to time-varying and bounded unknown inputs. In [13], a new sliding mode observer design method based on a high gain approach was proposed in the case where the observer matching condition is not satisfied. The authors of the latter paper have proposed a method based on the idea of generating auxiliary outputs in order to relax the assumption 5. Inspired from this approach, the main contribution of this paper is to solve the problem of adaptive observer design with a high gain approach despite that the observer matching condition 5 is not verified.

Definition 2.1. Let $C=\left[C_{1}, \ldots, C_{p}\right]^{T}$. The relative degree of the $i^{\text {th }}$ output $y_{i}=C_{i} x$ with respect to the unknown parameters vector $\theta$ is the smallest integer $r_{i}$ such that:

$C_{i} A^{m} B=0$, for $m=0, \ldots, r_{i}-2$ $C_{i} A^{r_{i}-1} B \neq 0$.

We assume that the observer matching condition 5 is not verified for System (1). That is, there exists at least an integer $j \in\{1, \ldots, p\}$ such that the relative degree $r_{j}$ of the $j^{\text {th }}$ output $y_{j}$ with respect to the unknown parameters vector $\theta$ is such that $r_{j} \geq 2$. We construct an auxiliary output vector $z=H x$ where the matrix

$$
H=\left[\begin{array}{c}
C_{1} \\
\vdots \\
C_{1} A^{q_{1}-1} \\
\vdots \\
C_{p} \\
\vdots \\
C_{p} A^{q_{p}-1}
\end{array}\right]
$$

is such that $\operatorname{Rank}(H B)=\operatorname{Rank}(B)$ where $q_{i}\left(1 \leq q_{i} \leq r_{i}\right)$ are appropriate integers to be chosen, for $i=1, \ldots, p$.

Remark 1. Note that if Assumption 4 is satisfied for the triple $(A, B, C)$, so the invariant zeros of the system model given by the triple $(A, B, H)$ are in the open left-hand complex plane. That is Assumption 4 is also satisfied for the triple $(A, B, H)$ - See [13] and [17].

Now, we will rather focus on the modified system based on the new generated auxiliary output vector :

$$
\left\{\begin{array}{l}
\dot{x}=A x+B f(x, u)+B g(x, u) \theta+E u \\
z=H x
\end{array}\right.
$$

Remark 2. To illustrate the procedure of generating auxiliary outputs, we consider again the twin rotor system (14) for which the assumption 5 is not satisfied. Let $C=\left[C_{1} ; C_{2}\right]$ with 
$C_{1}=\left[\begin{array}{llllll}1 & 0 & 0 & 0 & 0 & 0\end{array}\right]$ and $C_{2}=\left[\begin{array}{llllll}0 & 0 & 1 & 0 & 0 & 0\end{array}\right]$. We generate the auxiliary output as follows:

$$
z=H x=\left[\begin{array}{c}
z_{11}=C_{1} x \\
z_{12}=C_{1} A x \\
z_{12}=C_{2} x \\
z_{12}=C_{2} A x
\end{array}\right]
$$

where

$$
H=\left[\begin{array}{c}
C_{1} \\
C_{1} A \\
C_{2} \\
C_{2} A
\end{array}\right]=\left[\begin{array}{llllll}
1 & 0 & 0 & 0 & 0 & 0 \\
0 & 1 & 0 & 0 & 0 & 0 \\
0 & 0 & 1 & 0 & 0 & 0 \\
0 & 0 & 0 & 1 & 0 & 0
\end{array}\right]
$$

The observer matching condition for the twin rotor system (14) is now clearly satisfied by considering the new couple of matrices $(B, H)$ since $\operatorname{Rank}(H B)=\operatorname{Rank}(B)$.

Now we are ready to present our main result.

\section{Main result}

In this section, we propose a new adaptive estimation approach and we prove the convergence of the state estimation error as well as the parametric convergence based on a Lyapunov stability analysis. The proposed adaptive estimation approach is then extended to the case where the considered nonlinear systems are subject to time-varying disturbances and we improve the robustness of our approach inspired from the sliding modes theory.

\subsection{Estimation of the auxiliary outputs based on a high gain observer}

For the system (18), the observer matching condition is actually satisfied, however the auxiliary output $z=H x$ could not be used yet for the adaptive observer design because it is not available. To overcome this problem, we adopt the high gain observer which has been employed in [13] to generate an estimate $z_{h}$ for the auxiliary output $z=H x$. Let $z=\left[z_{1}^{T}, \ldots, z_{p}^{T}\right]^{T}$ with $z_{i}=\left[z_{i 1}, \ldots, z_{i q_{i}}\right]^{T}$ such that $z_{i j}=C_{i} A^{j-1} x$ for $i=1, \ldots, p$ and $j=1, \ldots, q_{i}$. If $q_{i}>1$, the dynamics of $z_{i}$ are given by

$$
\left\{\begin{array}{l}
\dot{z}_{i}=a_{i} z_{i}+b_{i 1} h_{i}(y, z, u, t, \theta)+b_{i 2} u \\
z_{i 1}=c_{i} z_{i}
\end{array}\right.
$$

where $\left(a_{i}, b_{i 1}\right)$ is in the canonical form, $h_{i}(y, z, u, t, \theta)=C_{i} A^{q_{i}} x+C_{i} A^{q_{i}-1} B[f(x, u)+$ $g(x, u) \theta], b_{i 2}=\left[C_{i} E \cdots C_{i} A^{q_{i}-1} E\right]$ and $c_{i}=[1,0, \ldots, 0]$. A high gain observer for the latter system is given by

$$
\left\{\begin{array}{l}
\dot{z}_{h i}=a_{i} z_{h i}+\Gamma_{i} c_{i}\left(z_{i}-z_{h i}\right)+b_{i 2} u \\
z_{h i 1}=c_{i} z_{h i}
\end{array}\right.
$$

where $z_{h i}=\left[z_{h i 1} \cdots z_{h i q_{i}}\right]^{T}$ and $\Gamma_{i}=\left[\frac{\gamma_{i 1}}{\varepsilon} \ldots \frac{\gamma_{i q i}}{\varepsilon^{q i}}\right]^{T} . \varepsilon$ is a design parameter to be chosen sufficiently small such that $\varepsilon \in(0,1)$ and $\gamma_{i j}$ (for $j=1, \ldots, q_{i}$ ) are selected such that the matrix 


$$
\bar{a}_{i}=\left[\begin{array}{cc}
-\gamma_{i}^{T} & I_{q_{i}-1} \\
-\gamma_{i} q_{i} & 0_{q_{i}-1}
\end{array}\right]
$$

is Hurwitz, where $\gamma_{i}=\left[\gamma_{i 1} \cdots \gamma_{i\left(q_{i}-1\right)}\right]-$ See [13] for further details.

Let $\zeta_{i}=\left[\zeta_{i 1} \ldots \zeta_{i q_{i}}\right]^{T}$ where

$$
\zeta_{i j}=\frac{z_{i j}-z_{h i j}}{\varepsilon^{q_{i}-j}}, j=1, \ldots, q_{i}
$$

Let now $\zeta=\left[\zeta_{1}^{T} \cdots \zeta_{p}^{T}\right]^{T}$ and $D=\operatorname{diag}\left[D_{1} \cdots D_{p}\right]$ such that $D_{i}=\operatorname{diag}\left[\varepsilon^{q_{i}-1} \varepsilon^{q_{i}-2} \cdots 1\right]$. Let $z_{h}=\left[z_{h 1}^{T} \cdots z_{h p}^{T}\right]^{T}$. So, we have

$$
z-z_{h}=D \zeta
$$

Using the arguments of [13], for the high gain observer (20), there exist a positive constant $\beta$ and a finite time $T(\varepsilon)$ such that for all $t \geq T(\varepsilon)$

$$
|\zeta(t)| \leq \beta \varepsilon
$$

Moreover, we have $\lim _{\varepsilon \rightarrow+\infty} T(\varepsilon)=0$. The obtained estimates of the auxiliary outputs are now ready to be used by the new adaptive observer that we propose next.

\subsection{Adaptive observer synthesis}

Using the estimated auxiliary output vector $z_{h}$ generated by the high gain observer (20) introduced in the previous section, the adaptive observer that we propose is described by the following dynamics:

$$
\dot{\hat{x}}=A \hat{x}+B f(\hat{x}, u)+B g(\hat{x}, u) \hat{\theta}+E u+\bar{L}\left(z_{h}-H \hat{x}\right)
$$

where $\hat{\theta}$ is an adaptive parameter updated online following the adaptation law:

$$
\dot{\hat{\theta}}=\delta g(\hat{x}, u)^{T} \bar{M}\left(z_{h}-H \hat{x}\right)-\sigma \hat{\theta}
$$

The estimated state is $\hat{x}$. The constants $\delta$ and $\sigma$ are positive real numbers imposed by the designer. The design matrices $\bar{L}$ and $\bar{M}$ are regular matrices of appropriate dimensions such that for some symmetric positive definite matrices $\bar{P}$ and $\bar{Q}$, we have

$$
\begin{gathered}
(A-\bar{L} H)^{T} \bar{P}+\bar{P}(A-\bar{L} H)=-2 \bar{Q} \\
B^{T} \bar{P}=\bar{M} H \\
|B|\left(K_{f}+K_{g} K_{\theta}\right)<\frac{\lambda_{\min }(\bar{Q})}{\lambda_{\max }(\bar{P})}
\end{gathered}
$$

Let $e:=x-\hat{x}$ and $\tilde{\theta}:=\theta-\hat{\theta}$. 
Differentiating on both sides of the latter, we obtain

$$
\begin{aligned}
\dot{e}= & A e+B f(x, u)+B g(x, u) \theta(t) \\
& -B f(\hat{x}, u)-B g(\hat{x}, u) \hat{\theta}(t) \\
& -\bar{L}\left(z_{h}-H \hat{x}\right) .
\end{aligned}
$$

Using (22), we get

$$
\begin{gathered}
\dot{e}=(A-\bar{L} H) e+B[f(x, u)-f(\hat{x}, u)] \\
+B[g(x, u) \theta(t)-g(\hat{x}, u) \hat{\theta}]+\bar{L} D \zeta
\end{gathered}
$$

Adding $B g(\hat{x}, u) \tilde{\theta}(t)$ to both sides of (30) we obtain

$$
\begin{aligned}
\dot{e}= & (A-\bar{L} H) e+B[f(x, u)-f(\hat{x}, u)] \\
& +B[g(x, u)-g(\hat{x}, u)] \theta(t) \\
& +B g(\hat{x}, u) \tilde{\theta}+\bar{L} D \zeta
\end{aligned}
$$

where, according to the adaptation law (25) and since $\dot{\theta}(t) \simeq 0$ (see Assumption 2), $\tilde{\theta}$ is solution to

$$
\begin{aligned}
\dot{\tilde{\theta}} & =-\delta g(\hat{x}, u)^{T} \bar{M}(H e-D \zeta)+\sigma \hat{\theta} \\
& =-\delta g(\hat{x}, u)^{T} \bar{M}(H e-D \zeta)-\sigma \tilde{\theta}+\sigma \theta
\end{aligned}
$$

where we have also used the equation (22).

Next, consider the positive definite Lyapunov function

$$
V(e, \tilde{\theta}):=e^{T} \bar{P} e+\frac{1}{\delta} \tilde{\theta}^{T} \tilde{\theta}
$$

Theorem 3.1. Consider the nonlinear uncertain system (1) under assumptions 2, 4, 6 and 7. Consider the adaptive observer (24) with adaptation law (25) and the high gain observer (20). Then, there exist positive constants $K_{0}, K_{1}, K_{2}$ et $K_{3}$ such that the state estimation error $e(t)$ and the adaptation error $\tilde{\theta}(t)$ are uniformly bounded and converge in a finite time $T_{f}(\varepsilon)$ to the compact set:

$$
D_{R}=\left\{e, \tilde{\theta}: V(e, \tilde{\theta})<\frac{\left(K_{2} \varepsilon+K_{3}\right)^{2}}{\left(K_{0}-K_{1} \varepsilon\right)^{2}}\right\}
$$

with a rate at least as fast as $e^{-K_{0}(t-T(\varepsilon))}$. 
Proof. The total time derivative of (33) along the trajectories of (31)-(32) yields

$$
\begin{aligned}
\dot{V} \quad & =e^{\top}\left[(A-\bar{L} H)^{\top} \bar{P}+\bar{p}(A-\bar{L} H)\right] e \\
& +2 e^{\top} \bar{P} B[f(x, u)-f(\hat{x}, u)] \\
& +2 e^{\top} \bar{P} B[g(x, u)-g(\hat{x}, u)] \theta(t) \\
& +2 e^{\top} \bar{P} B \tilde{\theta} g(\hat{x}, u)+2 e^{\top} \bar{P} \bar{L} D \zeta \\
& -2 \tilde{\theta}^{\top}\left[g(\hat{x}, u)^{\top} \bar{M} H e\right]-\frac{2 \sigma}{\delta} \tilde{\theta}^{2}+\frac{2 \sigma}{\delta} \theta \tilde{\theta} \\
& -2 \tilde{\theta}^{T}[g(x, u)-g(\hat{x}, u)]^{T} \bar{M} D \zeta \\
& +2 \tilde{\theta}^{T}[g(x, u)]^{T} \bar{M} D \zeta .
\end{aligned}
$$

We recall that from Assumption 6, the nonlinear function $g$ is once continuously differentiable and using the boundedness of the trajectroies of the considered system (Assumption 7), we can find a positive constant $G_{\max }$ such that for all $t \geq 0$, we have:

$$
|g(x, u)| \leq G_{\max }
$$

Then, using the equations (11), (12), (26), (27) and (34), we have

$$
\begin{aligned}
\dot{V} \leq & -2 e^{\top} \bar{Q} e+2\left|B^{T} \bar{P} e\right|\left[K_{f}+K_{g} K_{\theta}\right]|e| \\
& -\frac{2 \sigma}{\delta} \tilde{\theta}^{2}+2|\bar{P} \bar{L}||D||e||\zeta|+\frac{2 \sigma}{\delta} K_{\theta} \tilde{\theta} \\
& +2 G_{\max }|\bar{M}||D||\tilde{\theta}||\zeta|+2 K_{g}|\bar{M}||D||\tilde{\theta}||e||\zeta|
\end{aligned}
$$

Let $\tau:=\frac{\lambda_{\min }(Q)}{\lambda_{\max }(\bar{P})}-|B|\left[K_{f}+K_{g} K_{\theta}\right]$. Note also, from the definition of the matrix $D$ in the previous section, that its induced euclidean norm is equal to 1 , that is $|D|=1$. It follows that

$$
\begin{aligned}
\dot{V} \leq & -2 \tau e^{\top} \bar{P} e-\frac{2 \sigma}{\delta} \tilde{\theta}^{2}+2 \beta|\bar{P} \bar{L}| \varepsilon|e| \\
& +\left(2 \beta G_{\max }|\bar{M}| \varepsilon+\frac{2 \sigma}{\delta} K_{\theta}\right)|\tilde{\theta}|+2 \beta K_{g}|\bar{M}| \varepsilon|\tilde{\theta}||e|
\end{aligned}
$$

where we have also used the inequality (23). Let us now apply the Young's inequality on the term $|\tilde{\theta}||e|$ :

$$
\begin{aligned}
|\tilde{\theta}||e| & \leq \frac{1}{2}|\tilde{\theta}|^{2}+\frac{1}{2}|e|^{2} \\
& \leq \frac{1}{2 \lambda_{\min }(\bar{P})} e^{\top} \bar{P} e+\frac{\delta}{2}\left(\frac{1}{\delta} \tilde{\theta}^{T} \theta\right) \\
& \leq \max \left(\frac{1}{2 \lambda_{\min }(\bar{P})}, \frac{\delta}{2}\right) V .
\end{aligned}
$$


On the other hand, we have $|e| \leq \frac{\sqrt{V}}{\sqrt{\lambda_{\min }(\bar{P})}}$ and $|\tilde{\theta}| \leq \sqrt{\delta} \sqrt{V}$. So, it follows that:

$$
\dot{V} \leq-2 K_{0} V+K_{1} \varepsilon V+\left(K_{2} \varepsilon+K_{3}\right) \sqrt{V}
$$

where

$$
\begin{aligned}
K_{0} & =\min (\tau, \sigma) \\
K_{1} & =2 \beta \max \left(\frac{1}{2 \lambda_{\min }(\bar{P})}, \frac{\delta}{2}\right) K_{g}|\bar{M}| \\
K_{2} & =\frac{2 \beta|\bar{P} \bar{L}|}{\sqrt{\lambda_{\min }(\bar{P})}}+\sqrt{\delta} \beta G_{\max }|\bar{M}| \\
K_{3} & =\frac{2 \sigma}{\sqrt{\delta}} K_{\theta} .
\end{aligned}
$$

It follows that

$$
\dot{V} \leq-K_{0} V-\sqrt{V}\left[\left(K_{0}-K_{1} \varepsilon\right)-\left(K_{2} \varepsilon+K_{3}\right) \sqrt{V}\right] .
$$

We deduce that for all $t \geq T(\varepsilon)$, as long as $\left(K_{0}-K_{1} \varepsilon\right)-\left(K_{2} \varepsilon+K_{3}\right) \sqrt{V(e(t), \tilde{\theta}(t))}>0$, then $V(e(t), \tilde{\theta}(t))>R$ with $R=\frac{\left(K_{2} \varepsilon+K_{3}\right)^{2}}{\left(K_{0}-K_{1} \varepsilon\right)^{2}}$, we have $\dot{V}(e(t), \tilde{\theta}(t)) \leq-K_{0} V(e(t), \tilde{\theta}(t))$ and if $V(T(\varepsilon))>R, V(e(t), \tilde{\theta}(t))$ will be decreasing exponentially fast such that

$$
V(e(t), \tilde{\theta}(t)) \leq V(T(\varepsilon)) e^{-K_{0}(t-T(\varepsilon))} .
$$

Consequently, $e(t)$ and $\tilde{\boldsymbol{\theta}}(t)$ converge to the compact set:

$$
D_{R}=\left\{e, \tilde{\theta}: V(e, \tilde{\theta})<\frac{\left(K_{2} \varepsilon+K_{3}\right)^{2}}{\left(K_{0}-K_{1} \varepsilon\right)^{2}}\right\}
$$

Note that, from the equation (37), the convergence of $e(t)$ and $\tilde{\theta}(t)$ is ensured in a finite time:

$$
T_{f}(\varepsilon)=T(\varepsilon)+K_{0}^{-1} \operatorname{Ln}\left(\frac{V(T(\varepsilon))}{R}\right)
$$

which completes the proof.

Remark 3. The compact set $D_{R}$ may be reduced by choosing appropriately the design parameters $\varepsilon, \delta$ and $\sigma$.Two procedures may be used for the choice of the design parameters.

Procedure 1: For any fixed $\sigma$ and any fixed $\delta$ chosen sufficiently large $(\delta>>1$ and $\delta>>\sigma)$, we can always find a constant $\varepsilon$ sufficiently small $(\varepsilon<<1)$ to reduce the radius $R$ of the compact set $D_{R}$ :

Indeed, in this case, we start by fixing $\delta$ and $\sigma$ such that $\delta>>1$ and $\delta>>\sigma$ in 
such away that $K_{1}, K_{2}$ and $K_{3}$ are approximated by the following expressions:

$$
\begin{array}{r}
K_{1} \approx \beta \lambda \delta K_{g}|\bar{M}| \\
K_{2} \approx \sqrt{\delta} \beta G_{\max }|\bar{M}| \\
K_{3} \approx 0 .
\end{array}
$$

As a consequence, the radius $R$ of the compact set $D_{R}$ is approximated by:

$$
R \approx \frac{\left(\sqrt{\delta} \beta G_{\max }|\bar{M}| \varepsilon\right)^{2}}{\left(K_{0}-\beta \lambda \delta K_{g}|\bar{M}| \varepsilon\right)^{2}}
$$

By considering the latter expression, $\delta$ being fixed, it is clear that we can always find a sufficiently small $\varepsilon$ such that the radius $R$ of the compact set becomes negligible.

Procedure 2: For any fixed $\varepsilon$ and $\sigma$, the compact set $D_{R}$ may be reduced by choosing $\delta$ sufficiently large:

Indeed, we start by fixing arbitrary values of $\sigma$ and $0<\varepsilon<1$. Then, proceeding as in the procedure 1 , by choosing a sufficiently large value of $\delta$, we may deduce that the radius $R$ of the compact set $D_{R}$ is approximated by the expression (40). $\varepsilon$ and $\sigma$ being fixed, the radius $R$ of the compact set may be made as small as possible for sufficiently large values of $\delta$, since the numerator of the latter expression of $R$ depends on $\sqrt{\delta}$ whereas the denominator is function of $\delta$.

Remark 4. For the design of the adaptation law (25), we have used the $\sigma$-modification technique by adding the term $-\sigma \hat{\theta}$. The $\sigma$-modification technique is a classical solution in adaptive control to improve the robustness of adaptation laws - See for instance [18]. It is clear from the proof of our main theorem (3.1), that the term $-\sigma \hat{\theta}$ has played a principal role to ensure the convergence of both the states and the unknown parameters estimation errors to the compact set (38) independent to whether the persistency of excitation assumption 3 is satisfied or not. The persistency of excitation condition is a classical assumption usually employed in the literature of adaptive control. Assumption 3 leads to the statement of necessary and sufficient conditions for asymptotic stability and parameter convergence. It represents a strong hypothesis and its verification in practice constitutes a very hard task in many applications. In our adaptive estimation approach, by applying the $\sigma$-modification technique, we have the advantage to avoid this restrictive assumption: of course, we don't obtain the asymptotic stability in this case, however we get, as it was shown in Theorem 3.1, the very satisfactory result that the state estimation and adaptation errors converge in a finite time to a compact set whose the radius may be arbitrarily reduced.

\subsection{Robustness improvement of the proposed adaptive observer}

In this section, we investigate the case where the considered nonlinear system (1) is corrupted by additive disturbances and the dynamics of the perturbed system is given by:

$$
\left\{\begin{array}{l}
\dot{x}=A x+B f_{0}(x, u)+B g_{0}(x, u) \theta+E u+B v \\
y=C x
\end{array}\right.
$$

where $v(t)$ represents the time-varying unknown disturbances which are assumed to be 
uniformly bounded and there exists a positive constant $K_{V}$ such that $|v(t)| \leq K_{v}$.

Proceeding as in the adaptive estimation approach developed above, after applying the Lipschitz extension technique (Refer to Subsection 2.2) and the procedure of generating auxiliary outputs (see Subsection 2.2.2), we obtain the following modified system:

$$
\left\{\begin{array}{l}
\dot{x}=A x+B f(x, u)+B g(x, u) \theta+E u+B v \\
z=H x
\end{array}\right.
$$

where $f$ and $g$ are the prolongations of the nonlinear functions $f_{0}$ and $g_{0}$ and satisfy the Lipschitz condition whith Lipschiz constants $K_{f}$ and $K_{g} . H$ is the augmented matrix obtained in Subsection 2.2.2.

The improved adaptive observer that we propose is given by the following dynamics

$$
\dot{\hat{x}}=A \hat{x}+B f(\hat{x}, u)+B g(\hat{x}, u) \hat{\theta}+E u+B U_{s}+\bar{L}\left(z_{h}-H \hat{x}\right)
$$

for which we associate the adaptation law (25), the high gain observer (20) and the discontinuous input $U_{s}$ given by

$$
U_{s}= \begin{cases}K_{s} \frac{\bar{M}\left(z_{h}-H \hat{x}\right)}{\left|\bar{M}\left(z_{h}-H \hat{x}\right)\right|} & \text { if } \bar{M}\left(z_{h}-H \hat{x}\right) \neq 0 \\ 0 & \text { if } \bar{M}\left(z_{h}-H \hat{x}\right)=0\end{cases}
$$

whose the objective is to compensate for the additive disturbance $v(t)$. Next, the dynamics of the state estimation error $e=x-\hat{x}$ is given by

$$
\begin{gathered}
\dot{e}=(A-\bar{L} H) e+B[f(x, u)-f(\hat{x}, u)] \\
+B[g(x, u)-g(\hat{x}, u)] \theta(t) \\
+B g(\hat{x}, u) \tilde{\theta}+B\left[v-U_{s}\right]+\bar{L} D \zeta
\end{gathered}
$$

Theorem 3.2. Consider the nonlinear uncertain system (41) under assumptions 2, 4, 6 and 7. Consider the robust adaptive observer (43) with the discontinuous input (44), the adaptation law (25) and the high gain observer (20). Then, there exists positive constants $K_{1}, K_{2}, K_{4}$ and $K_{5}$ such that the state estimation error $e(t)$ and the adaptation error $\tilde{\theta}(t)$ are uniformly bounded and converge in a finite time $T_{f}(\varepsilon)$ to the compact set:

$$
D_{\Omega}=\left\{e, \tilde{\theta}: V<\Omega_{+}^{2}\right\}
$$

where

$$
\Omega_{+}=\frac{\left(K_{2} \varepsilon+K_{3}\right)^{2}+\sqrt{\left(K_{2} \varepsilon+K_{3}\right)^{2}+4 K_{4} K_{5} \varepsilon}}{2 K_{5}}
$$

with a rate at least as fast as $e^{-K_{0}(t-T(\varepsilon))}$.

Proof. We consider again the positive definite Lyapunov function (33). 
Following the same steps of the proof of Theorem 3.1, we deduce that the total time derivative of (33) along the trajectories of the system of equations (32) and (45), satisfies the following inequality

$$
\begin{aligned}
\dot{V} \leq & -2 K_{0} V+K_{1} \varepsilon V+\left(K_{2} \varepsilon+K_{3}\right) \sqrt{V} \\
& +2 e^{T} \bar{P} B v-2 e^{T} \bar{P} B U_{s}
\end{aligned}
$$

Now, replacing $U_{s}$ by its expression (44), we obtain

$$
\begin{aligned}
\dot{V} \leq & -2 K_{0} V+K_{1} \varepsilon V+\left(K_{2} \varepsilon+K_{3}\right) \sqrt{V} \\
& +2(\bar{M} H e+\bar{M} D \zeta)^{T} v \\
& -2 K_{s}|\bar{M} H e+\bar{M} D \zeta| \\
& +2(\bar{M} D \zeta)^{T}\left(U_{s}-v\right) .
\end{aligned}
$$

It follows that

$$
\begin{aligned}
\dot{V} \leq & -2 K_{0} V+K_{1} \varepsilon V+\left(K_{2} \varepsilon+K_{3}\right) \sqrt{V} \\
& -2\left(K_{s}-K_{v}\right)|\bar{M} H e+\bar{M} D \zeta| \\
& +2\left(K_{V}+K_{s}\right)|\bar{M}||D||\zeta|
\end{aligned}
$$

then, using the inequality (23) ant that $|D|=1$ as it was explained in the proof of Theorem 3.1 , we get

$$
\dot{V} \leq-2 K_{0} V+K_{1} \varepsilon V+\left(K_{2} \varepsilon+K_{3}\right) \sqrt{V}+K_{4} \varepsilon
$$

where $K_{4}=2\left(K_{v}+K_{s}\right) \beta|\bar{M}|$.

It follows that

$$
\dot{V} \leq-K_{0} V-\left(K_{5} V-\left(K_{2} \varepsilon+K_{3}\right) \sqrt{V}-K_{4} \varepsilon\right)
$$

where $K_{5}=K_{0}-K_{1} \varepsilon$. We can choose the design parameter $\varepsilon$ sufficiently small and there exists $\varepsilon^{*} \in[0,1]$ such that for all $\varepsilon<\varepsilon^{*}$, we have $K_{5}=K_{0}-K_{1} \varepsilon>0$. It follows that for all $\varepsilon<\varepsilon^{*}$, we have

$$
\dot{V} \leq-K_{0} V-K_{5}\left(\sqrt{V}-\Omega_{-}\right)\left(\sqrt{V}-\Omega_{+}\right)
$$


where

$$
\begin{gathered}
\Omega_{-}=\frac{\left(K_{2} \varepsilon+K_{3}\right)^{2}-\sqrt{\left(K_{2} \varepsilon+K_{3}\right)^{2}+4 K_{4} K_{5} \varepsilon}}{2 K_{5}}<0 \\
\Omega_{+}=\frac{\left(K_{2} \varepsilon+K_{3}\right)^{2}+\sqrt{\left(K_{2} \varepsilon+K_{3}\right)^{2}+4 K_{4} K_{5} \varepsilon}}{2 K_{5}}>0 .
\end{gathered}
$$

We deduce that for all $t \geq T(\varepsilon)$, as long as $\sqrt{V(e(t), \tilde{\theta}(t))}>\Omega_{+}$, that is $V(e(t), \tilde{\theta}(t))>$ $\Omega_{+}^{2}$, we have $K_{5}\left(\sqrt{V(e(t), \tilde{\theta}(t))}-\Omega_{-}\right)\left(\sqrt{V(e(t), \tilde{\theta}(t))}-\Omega_{+}\right)>0$ and consequently $\dot{V}(e(t), \tilde{\theta}(t)) \leq-K_{0} V(e(t), \tilde{\theta}(t))$ and if $V(T(\varepsilon))>\Omega_{+}^{2}, V(e(t), \tilde{\theta}(t))$ will be decreasing exponentially fast such that

$$
V(e(t), \tilde{\theta}(t)) \leq V(T(\varepsilon)) e^{-K_{0}(t-T(\varepsilon))} .
$$

Consequently, $e(t)$ and $\tilde{\boldsymbol{\theta}}(t)$ converge to the compact set:

$$
D_{\Omega}=\left\{e, \tilde{\theta}: V(e, \tilde{\theta})<\Omega_{+}^{2}\right\}
$$

Note that from equation (58), the convergence of $e(t)$ and $\tilde{\theta}(t)$ is ensured in a finite time:

$$
T_{f}(\varepsilon)=T(\varepsilon)+K_{0}^{-1} \operatorname{Ln}\left(\frac{V(T(\varepsilon))}{\Omega+^{2}}\right)
$$

which completes the proof.

\section{Numerical simulations}

In order to illustrate the effectiveness of the proposed high gain observer based adaptive estimation approach, we consider again the nonlinear system consisting in a twin rotor MIMO system described by the state model (14). The numerical values of the physical parameters of the twin rotor system are given in the table below. 


\begin{tabular}{|l|l|}
\hline Physical parameters & Numerical values \\
\hline$I_{1}:$ Moment of inertia of vertical rotor & $0.068 \mathrm{Kg} \cdot \mathrm{m}^{2}$ \\
\hline$I_{2}:$ Moment of inertia of horizontal rotor & $0.002 \mathrm{Kg} \cdot \mathrm{m}^{2}$ \\
\hline$a_{1}:$ Static characteristic & 0.0135 \\
\hline$b_{1}:$ Static characteristic & 0.0924 \\
\hline$a_{2}:$ Static characteristic & 0.02 \\
\hline$b_{2}:$ Static characteristic & 0.09 \\
\hline$M_{g}:$ Gravity momentum & $0.32 \mathrm{~N} . \mathrm{m}$ \\
\hline$B_{1 \alpha_{v}}:$ Friction momentum function & $0.006 \mathrm{~N} . \mathrm{m} . \mathrm{s} / \mathrm{rad}$ \\
\hline$B_{1 \alpha_{h}}:$ Friction momentum function & $0.1 \mathrm{~N} . \mathrm{m} . \mathrm{s} / \mathrm{rad}$ \\
\hline$k_{g y}:$ Gyroscopic momentum parameter & $0.05 \mathrm{~s} / \mathrm{rad}$ \\
\hline$k_{1}:$ Main rotor gain & 1.1 \\
\hline$k_{2}:$ Tail rotor gain & 0.8 \\
\hline$T_{11}:$ Main rotor denominator & 1.1 \\
\hline$T_{10}:$ Main rotor denominator & 1 \\
\hline$T_{21}:$ Tail rotor denominator & 1 \\
\hline$T_{20}:$ Tail rotor denominator & 1 \\
\hline$T_{p}:$ Cross react. momentum parameter & 2 \\
\hline$T_{0}:$ Cross react. momentum parameter & 3.5 \\
\hline$k_{c}:$ Cross reaction momentum gain & -0.2 \\
\hline
\end{tabular}

The control inputs are taken as $u_{1}=u_{v}=\sin (t)$ and $u_{2}=u_{h}=0.5 \sin (5 t)$. The initial conditions of the twin rotor state model are selected as $x(0)=[0.2,0.4,0.6,0.8,1,1.2]^{T}$.

Under these conditions, the trajectories of the state model (14) are bounded. The nonlinear function $f(x, u)=\left[f_{1}(x, u) ; f_{2}(x, u)\right]$ is clearly continuously differentiable. As a consequence, we can apply the Lipschitz-extension technique by following the procedure presented in Subsection 2.2.

Let $X=\left\{x \in \mathbb{R}^{n},\left|x_{i}\right| \leq d_{i}, i=1, \cdots, 6\right\}$. The saturation levels are set to $d_{1}=d_{2}=\cdots=$ $d_{6}=5$. Let $\eta(x)$ be a linear saturation such that $\eta(x)=\left[\eta_{1}(x), \eta_{2}(x), \cdots, \eta_{6}(x)\right]^{T}$ and for $i=1 \cdots 6$,

$$
\eta_{i}(x)=\left\{\begin{array}{c}
d_{i} \quad \text { if } \quad x_{i}>d_{i} \\
x_{i} \text { if }-d_{i} \leq x_{i} \leq d_{i} \\
-d_{i} \text { if } \quad x_{i}>d_{i}
\end{array}\right.
$$

Next, we define the prolongations $f(x, u)=\left[f_{1}(x, u, t), f_{2}(x, u, t)\right]^{T}$ and $g(x, u)=$ $\left[g_{1}(x, u), 0\right]^{T}$ of the nonlinear functions $f(x, u)$ and $g(x, u)$ as follows

$$
\begin{aligned}
f_{1}(x, u, t)= & \frac{a_{1}}{I_{1}}\left(\eta_{5}\left(x_{5}\right)\right)^{2}+\frac{0.0326}{2 I_{1}} \sin \left(2 \eta_{2}\left(x_{2}\right)\right)\left(\eta_{4}\left(x_{4}\right)\right)^{2} \\
& -\frac{k_{g} y}{I_{1}} a_{1} \cos \left(\eta_{1}\left(x_{1}\right)\right) \eta_{4}\left(x_{4}\right)\left(\eta_{5}\left(x_{5}\right)\right)^{2} \\
& -\frac{k_{g y}}{I_{1}} b_{1} \cos \left(\eta_{1}\left(x_{1}\right)\right) \eta_{4}\left(x_{4}\right) \eta_{5}\left(x_{5}\right) \\
f_{2}(x, u, t)= & \frac{a_{2}}{I_{2}}\left(\eta_{6}\left(x_{6}\right)\right)^{2}-\frac{k_{c} a_{1}}{I_{2}} 1.75\left(\eta_{5}\left(x_{5}\right)\right)^{2}
\end{aligned}
$$




$$
g_{1}(x, u, t)=-\frac{1}{I_{1}} \sin \left(\eta_{1}\left(x_{1}\right)\right)
$$

Under these conditions, for all $x \in X, f(x, u)=f_{0}(\eta(x), u, t)$ and $g(x, u)=g_{0}(\eta(x), u, t)$. Moreover, the extended nonlinear functions $f$ and $g$ are globally Lipschitz with respective Lipschitz constants $K_{f}$ and $K_{g}$. Hence, the twin rotor state model (14) can be written in the form (13) whose the trajectories coincide with those of System (1) for all $t$ such that $x(t) \in X$.

As it was mentioned in Section 2.2.1, the observer matching assumption 5 is not satisfied for the couple of matrices $(B, C)$ of the twin rotor system. We recall that the generated auxiliary output is given by: (see Remark 2 )

$$
z=\left[\begin{array}{c}
z_{11}=C_{1} x \\
z_{12}=C_{1} A x \\
z_{12}=C_{2} x \\
z_{12}=C_{2} A x
\end{array}\right]
$$

Since $C_{1} B=C_{2} B=0$, we have, for $i \in\{1,2\}$

$$
\left\{\begin{array}{l}
\dot{z}_{i 1}=C_{i} \dot{x}=C_{i} A x=z_{i 2} \\
\dot{z}_{i 2}=C_{i} A \dot{x}=C_{i} A^{2} x+C_{i} A B[f(x, u)+g(x, u) \theta] \\
z_{i 1}=c_{i} z_{i}
\end{array}\right.
$$

which is of the form of (19) with $h_{i}(z, y, u, t)=C_{i} A^{2} x+C_{i} A B[f(x, u)+g(x, u) \theta], b_{i 1}=[0,1]^{T}$, $c_{i}=\left[\begin{array}{ll}1 & 0\end{array}\right], b_{i 2}=\left[\begin{array}{lll}0 & 0 ; 0 & 0\end{array}\right]$ and

$$
a_{i}=\left[\begin{array}{ll}
0 & 1 \\
0 & 0
\end{array}\right]
$$

for $i \in\{1,2\}$. The high gain observer corresponding to system (65) is given by

$$
\left\{\begin{array}{l}
\dot{z}_{h i}=a_{i} z_{h i}+\Gamma_{i} c_{i}\left(z_{i 1}-z_{h i}\right) \\
z_{h i 1}=c_{i} z_{h i}
\end{array}\right.
$$

where $\Gamma_{i}=\left[\frac{\gamma_{i 1}}{\varepsilon}, \frac{\gamma_{i 2}}{\varepsilon^{2}}\right]^{T}$, for $i \in\{1,2\}$. We select $\gamma_{11}=\gamma_{21}=6, \gamma_{12}=\gamma_{22}=8$ and $\varepsilon=0.006$.

We design a robust adaptive observer of the form (24) with the adaptation law (25) and the high gain observer (20). The initial states of both high-gain and adaptive observers are set to zero and the adaptive parameter is initialized as $\hat{\theta}(0)=0$. The design parameters are set to $\varepsilon=0.006, \sigma=0.01, \delta=500$. To solve the system of linear matrix equations (26) and (27), one considers the following convex optimization problem [19]:

Minimize $\rho$ subject to

$$
\begin{aligned}
& \bar{P}>0 \\
& \bar{P} A-K H+(\bar{P} A-K H)^{T}<0 \\
& {\left[\begin{array}{cc}
\rho I & B^{T} \bar{P}-\bar{M} H \\
\left(B^{T} \bar{P}-\bar{M} H\right)^{T} & \rho I
\end{array}\right]>0}
\end{aligned}
$$


This problem may be solved by using the package $c v x$ specific to LMIs problems in association with MATLAB. When this optimization problem has a minimum $\rho=0$, the system of equations (26) and (27) is satisfied and numerical values of $\bar{P}, \bar{M}$, and $\bar{L}=\bar{P}^{-1} K$ may be obtained. The numerical values of the design matrices $\bar{M}$ and $\bar{L}$ are selected respectively as

$$
\begin{gathered}
\bar{M}=\left[\begin{array}{llll}
0 & 1 & 0 & 0 \\
0 & 0 & 0 & 1
\end{array}\right] \\
\bar{L}=\left[\begin{array}{cccc}
30 & 1 & 0 & 0 \\
0 & 29.05 & 0 & -0.185 \\
0 & 0 & 35 & 1 \\
0 & -2.0598 & 0 & -11.0475 \\
0 & 128.1885 & 0 & -1.6538 \\
0 & -46.593 & 0 & 8.045
\end{array}\right] .
\end{gathered}
$$

Firstly, tests are carried out without disturbances (i.e. with $v(t)=0)$ using the state model of the twin rotor (14) and the adaptive observer (24) with the adaptation law (25) and the high gain observer (20). The obtained simulation results are as follows. In Figures 1 and 2, we illustrate the convergence of the high gain observer (20) and the estimation of the auxiliary output $z_{h 12}$. Figure 3 illustrates the convergence of the the estimation errors of the proposed adaptive observer (24). The estimation of the states $x_{4}$ and $x_{5}$ by the adaptive observer (24) with the adaptation law (25) is illustrated in Figures 4 and 5, respectively. The reconstruction of the unknown parameter $\theta_{1}=M_{g}$ is shown in Figure 6 .

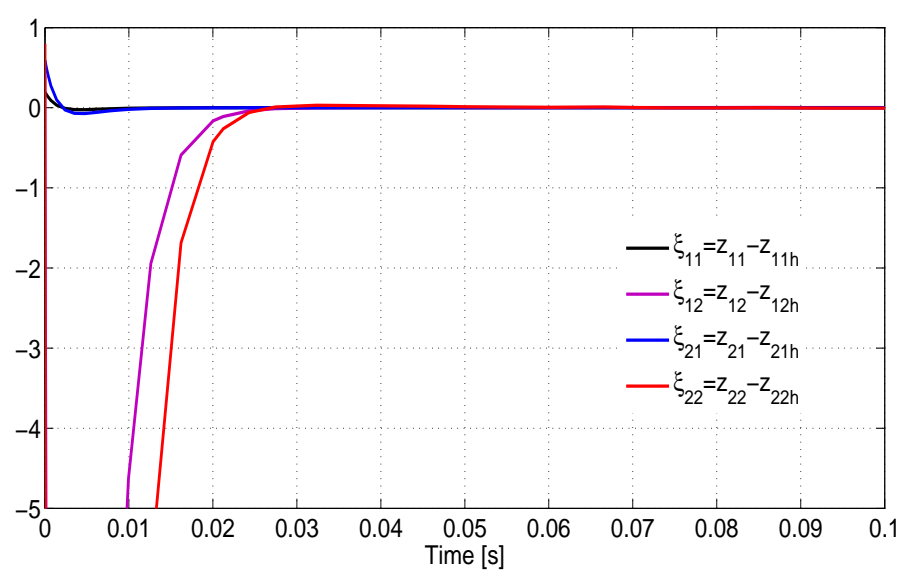

Figure 1. Evolution of the state estimation errors of the high gain observer (20) 


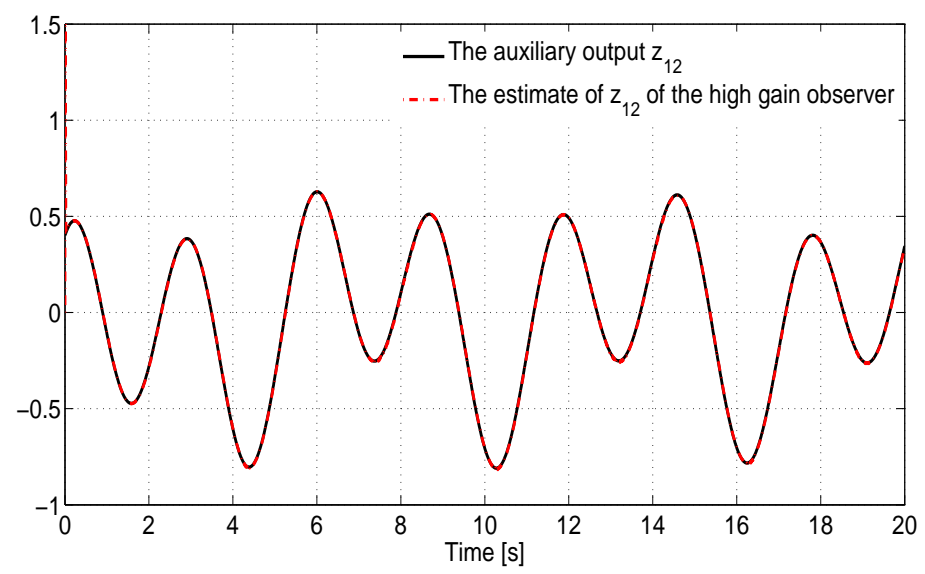

Figure 2. Evolution of the auxiliary output $z_{12}$ and its estimate $z_{12 h}$ by the high gain observer (20)

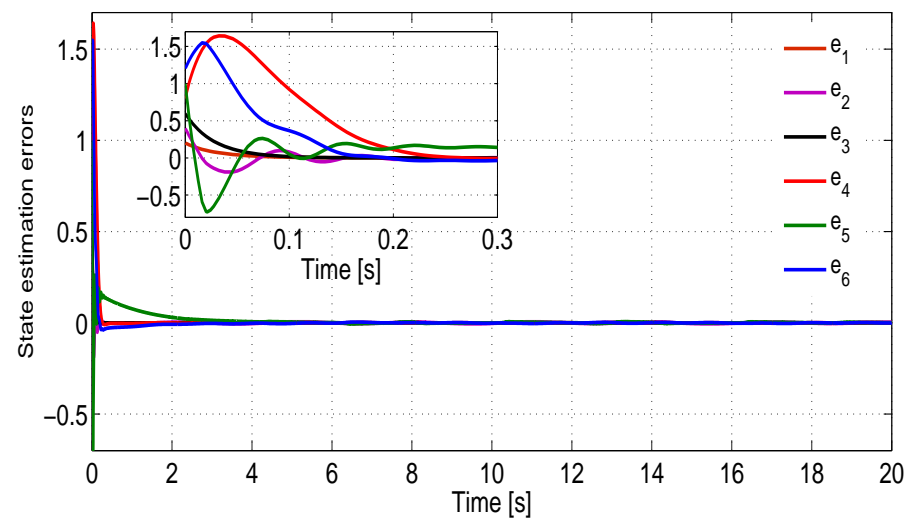

Figure 3. Evolution of the state estimation errors $e_{i}=x_{i}-\hat{x}_{i}$ of the adaptive observer (24) with the adaptation law (25) for $i=1, \cdots, 6$ in the absence of disturbances. 


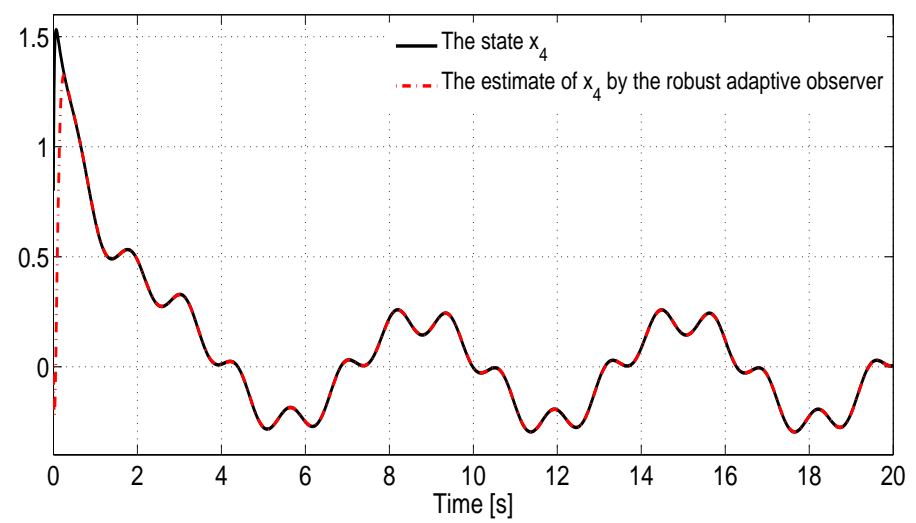

Figure 4. Evolution of the state $x_{4}$ and its estimate $\hat{x}_{4}$ by the adaptive observer (24) with the adaptation law (25) in the absence of disturbances.

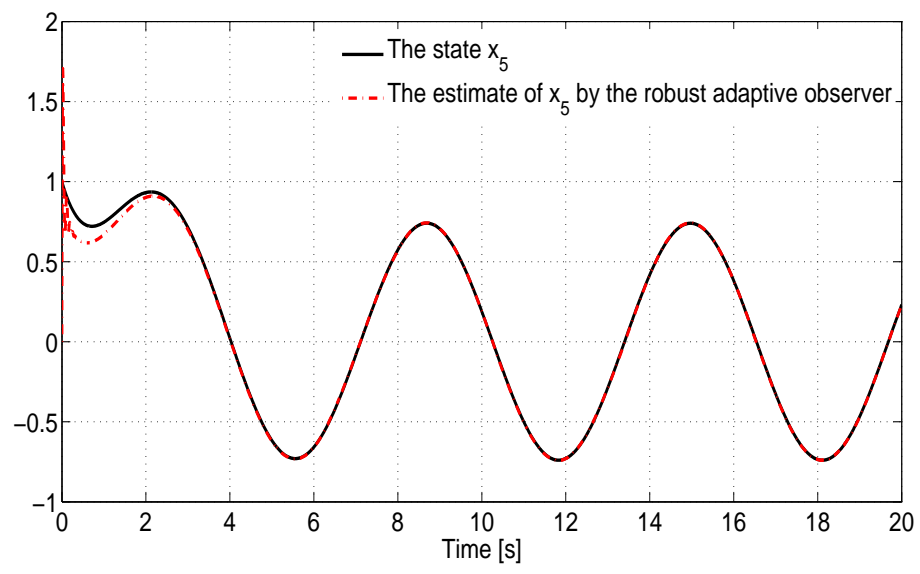

Figure 5. Evolution of the state $x_{5}$ and its estimate $\hat{x}_{5}$ by the adaptive observer (24) with the adaptation law (25) in the absence of disturbances. 


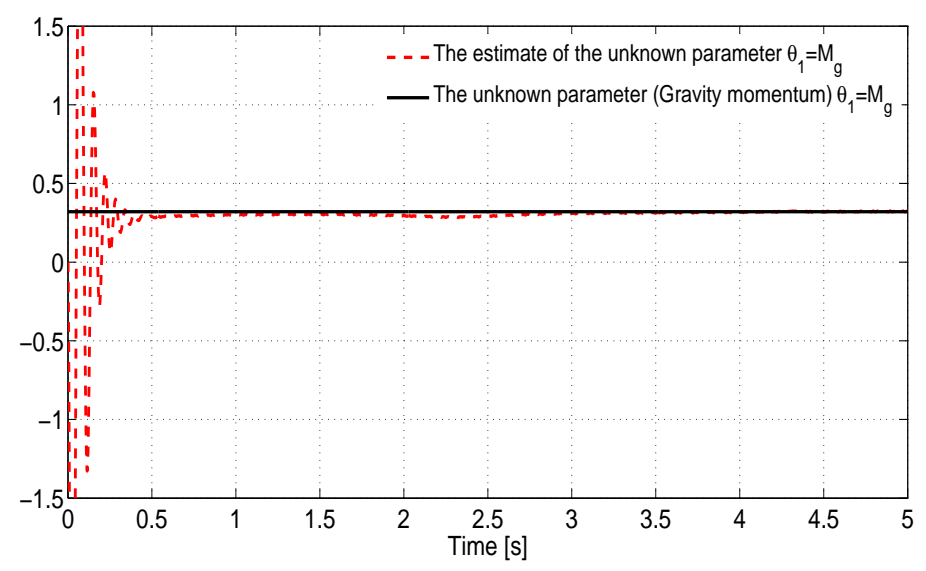

Figure 6. The unknown parameter $\theta_{1}=M_{g}$ (Gravity momentum) and its estimate $\hat{\theta}_{1}$ by the adaptive observer (24) with the adaptation law (25) in the absence of disturbances.

In a second run of simulations, the twin rotor system is corrupted by a disturbance signal $v(t)=2 h(t)$ where $h(t)$ is the unit step signal. The disturbance $v(t)$ appears in the second state equation (dynamics of the pitch angular velocity $x_{2}=\dot{\alpha}_{v}$ ). To illustrate the robustness of the improved robust estimation approach introduced in Subsection 3.3, we run simultaneously the adaptive observer (24) and the improved robust adaptive observer (43) for the perturbed twin rotor system. We select the design parameter $K_{s}=20$ for the discontinuous input (44). The robustness improvement in the presence of disturbances is shown in Figures 7 and 8. Indeed, it is clear from the latter figures that the improved robust adaptive observer (43) associated to the discontinuous input (44) possesses better performances in terms of robustness against perturbations compared to the adaptive observer (24).

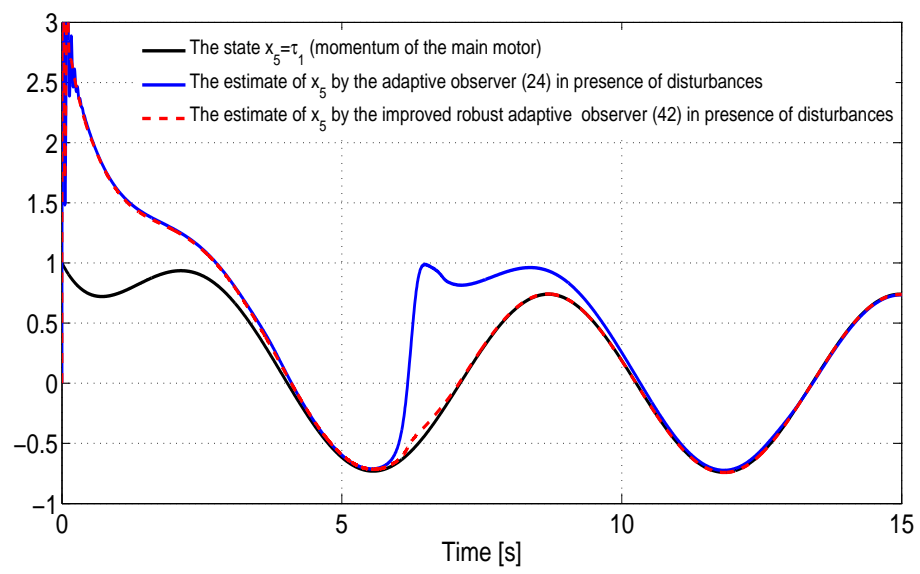

Figure 7. The estimates of the state $x_{5}$ by the adaptive observer (24) (Solid blue) and by the improved robust adaptive observer (43) with the discontinuous input (44) (Dashed red) in the presence of disturbances. 


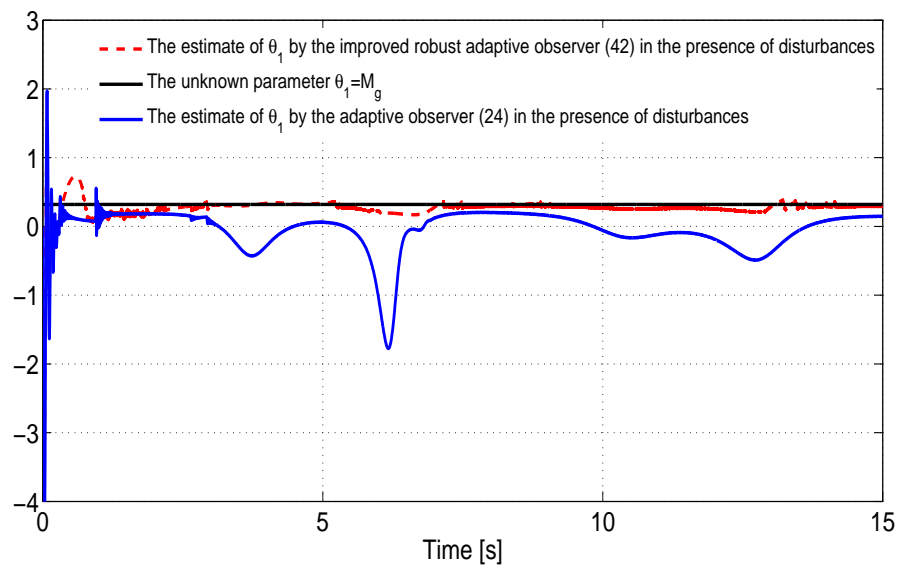

Figure 8. The estimates of the unknown parameter $\theta_{1}=M_{g}$ by the adaptive observer (24) (Solid blue) and by the improved robust adaptive observer (43) with the discontinuous input (44) (Dashed red) in the presence of constant disturbances.

In a more sophisticated scenario, additional constraints which resemble those occurring in real life applications are considered. Actually, we assume that the unknown parameter (the gravity momentum) is varying in such away that $\theta_{1}$ takes the following expression:

$$
\theta_{1}= \begin{cases}0.32 N m & \text { for } 0 \leq t<5 s \\ 2.32 N m & \text { for } 5 \leq t<10 s\end{cases}
$$

This configuration may be realized in practice by attaching an additional constant load to the counter balance of the twin rotor system at the middle of the experience since the gravity momentum depends on the mass of the counter balance.

In the actual scenario, we assume also that the dynamics of the twin rotor system is corrupted by time varying disturbances which may include unmodeled dynamics, external disturbances, actuator failures and process noise. That is, the disturbances signal $v(t)$ is actually given by

$$
v(t)=1.5 \sin (10 t)+1.75 \sin (5 t)+h(t-2)+w_{p}(t),
$$

where $h(t)$ is the unit step signal. The disturbance signal $h(t-2)$ represents a possibly actuator failure occurring at the time $t=2 s . w_{p}(t)$ is a zero mean Gaussian noise of variance equal to 0.02 .

All tests that will be presented below are realized with a varying unknown parameter $\theta_{1}$ given by (68) and a disturbances signal $v(t)$ given by expression (69).

In Figure 9, it is shown that the adaptive observer (24) and the improved observer (43) present satisfactory performances despite the presence of the disturbances signal (69), but it is also worth noticing that the robustness is considerably improved when using the improved observer (43). 


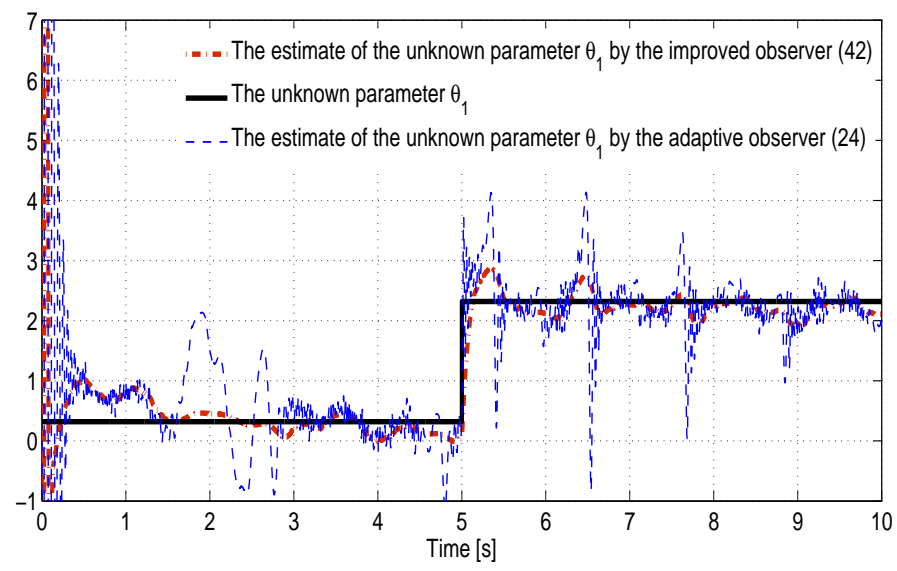

Figure 9. Estimation of a varying unknown parameter $\theta_{1}$ by the adaptive observer (24) (Dashed blue) and by the improved robust adaptive observer (43) with the discontinuous input (44) (Dashed red) in the presence of time varying disturbances and process noise with $\varepsilon=0.001, \delta=500$ and $\sigma=0.01$.

In a second test, the output signals are corrupted by a white noise of power equal to $10^{-9}$. It is well known that in such conditions, the measurement noise will be amplified by the high gain observer when choosing a relatively small value of the design parameter $\varepsilon$. To minimize this effect, we choose a larger value of $\varepsilon$ which actually takes a fixed value $\varepsilon=0.04$ (instead of 0.006 ). Next, following the procedure 2 presented in Remark 4 , we choose a larger value of $\delta(\delta=2000$ instead of 500) in order to reduce the amplitude of estimation and adaptation errors. As it is shown in Figure 10, the adaptive estimation presents some degradation compared to the measurement noise free case (See Figure 9). However, it is also to be noticed that with the new selected design parameters, the robustness against measurement noise is considerably improved compared to the case simulated with the old values of the design parameters in the presence of noisy measurements.

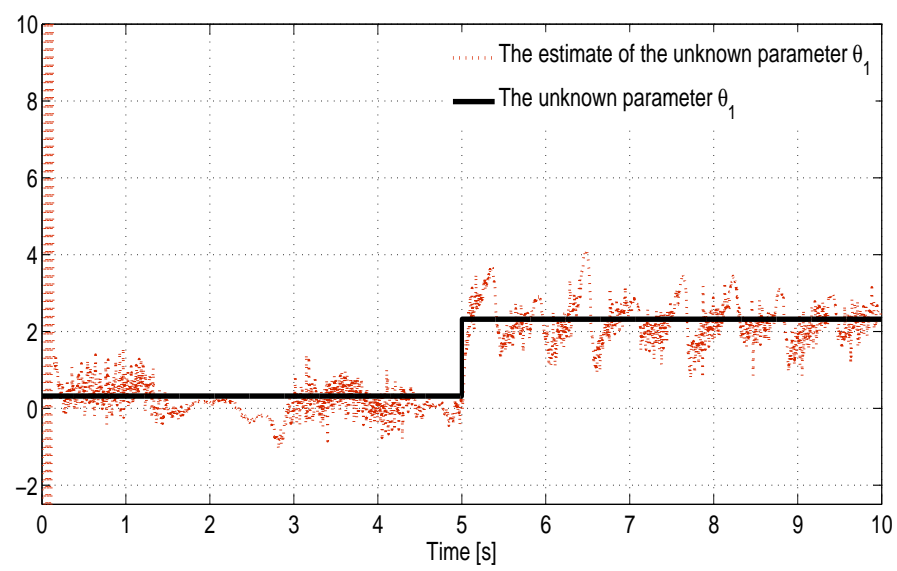

Figure 10. Estimation of a varying unknown parameter $\theta_{1}$ by the improved robust adaptive observer (43) with the discontinuous input (44) (Dashed red) in the presence of time varying disturbances, process noise and measurement noise with $\varepsilon=0.04$ $\delta=2000$ and $\sigma=0.01$.

Finally, measurement delays may also occur in practice. In Figure 11, the configuration simulated in the presence of a measurement delay $\tau_{d}$ equal to $100 \mathrm{~ms}$ depicts some degradation of the adaptive estimation performances.

It is worth noticing that in the literature of high gain observers, several observer design meth- 
ods which have been proposed to reduce the effect of noisy and delayed measurements, may be exploited for our adaptive estimation approach (See for instance the references [20], [21] for the noisy measurements case and the references [22], [23] for the delayed measurements case. Fundamental theoretical study of these problems as well as the experimental validation of our theoretical results will be investigated in our future works.

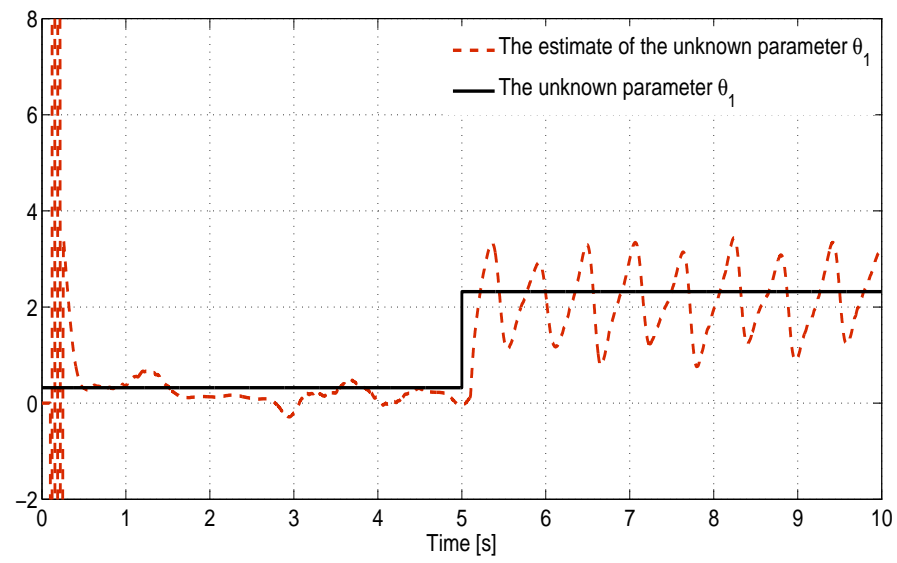

Figure 11. Estimation of a varying unknown parameter $\theta_{1}$ by the improved robust adaptive observer (43) with the discontinuous input (44) (Dashed red) in the presence of time varying disturbances and a measurement delay $\tau_{d}=100 \mathrm{~ms}$ with $\varepsilon=0.001$, $\delta=500$ and $\sigma=0.01$.

\section{Conclusion}

In this paper, we proposed a new adaptive estimation approach for a class of nonlinear systems subject to unknown parameters. The restrictive classical observer matching condition usually used in the literature of adaptive observer is rather weakened in this work by adopting the idea of generating auxiliary outputs which are estimated by an appropriate high gain observer. The estimated auxiliary outputs are employed by our proposed adaptive observer which ensures the both estimation error and parametric convergence to a compact set which may be reduced by appropriately choosing the design parameters. The robustness of the proposed adaptive observer to the unknown disturbances was improved by injecting a robust discontinuous term in its dynamics. Theoretical results where validated by some numerical simulations for the twin rotor MIMO system.

\section{References}

[1] Q. Zhang, "Adaptive observer for multiple-inputmultiple-output (mimo) linear timevarying systems," IEEE Trans. on Automat. Control, vol. 47, no. 3, pp. 525-529, 2002.

[2] J. D. L. Morales and G. B. andO. Huerta-Guevara, "On adaptive observers for state affine systems," International Journal of Control, vol. 79, pp. 581-591, 2006.

[3] T. Maatoug, M. Farza, M. M'Saad, Y. Koubaa, and M. Kamouni, "Adaptive output feedback controller for a class of uncertain nonlinear systems," in Proc. 17th IFAC world congress, (Seoul, Corea), pp. 13139-13144, 2008.

[4] M. Farza, M. MSaad, T. Maatoug, and M. Kamoun, "Adaptive observers for nonlinearly parameterized class of nonlinear systems," Automatica, pp. 2292-2299, 2009. 
[5] H. F. Grip, A. Saberi, and T. A. Johansen, "Estimation of states and parameters for linear systems with nonlinearly parameterized perturbations," Systems \& Control Letters, vol. 60, pp. 771-777, 2011.

[6] I. Y. Tyukin, E. Steur, H. Nijmeijer, and C. V. Leeuwen, "Adaptive observers and parameter estimation for a class of systems nonlinear in the parameters," Automatica, vol. 49, pp. 2409-2423, 2013.

[7] M. Farza, I. Bouraoui, T. Ménard, R. Abdennour, and M. M'Saad, “Adaptive observers for a class of uniformly observable systems with nonlinear parametrization and sampled outputs," Automatica, vol. 50, pp. 2951-2960, 2014.

[8] M. Farza, M. M'Saad, T. Ménard, A. Ltaief, and T. Maatoug, "Adaptive observer design for a class of nonlinear systems. application to speed sensorless induction motor," Automatica, vol. 90, pp. 239-247, 2018.

[9] A. loria, E. Panteley, and A. Zavala, "Adaptive observers for robust synchronization of chaotic systems," IEEE Trans. on Circ. Syst. I: Regular Papers, vol. 56, no. 12, pp. 27032716, 2009.

[10] D. Efimov, C. Edwards, and A. Zolghadri, "A note on improvement of adaptive observer robustness," in Proc. 19th IFAC world congress, (Cape Town, South Africa), pp. 558562, 2014.

[11] Y. M. Cho and R. Rajamani, "Systematic approach to adaptive observer synthesis for nonlinear systems," IEEE Trans. on Automat. Control, vol. 42, no. 4, pp. 534-537, 1997.

[12] G. Besançon, "Remarks on nonlinear adaptive observer design," Systems and Control Letters, vol. 41, pp. 271-280, 2000.

[13] K. Kalsi, J. Lian, S. Hui, and S. H. Zak, "Sliding-mode observers for systems with unknown inputs: A high-gain approach," Automatica, vol. 46, pp. 347-353, 2010.

[14] H. Shim, Y. I. Son, and J. H. Seo, "Semi-global observer for multi-output nonlinear systems," Systems and Control Letters, vol. 42, pp. 233-244, 2001.

[15] H. Dimassi and A. Loría, "Adaptive unknown-input observers-based synchronization of chaotic systems for telecommunication," IEEE Transactions on circuits and systems I, vol. 58, pp. 800-812, 2011.

[16] S. T. Hui and S. H. Zak, "Observer design for systems with unknown inputs," Int. J. Appl. Math. Comput. Sci., vol. 15, no. 4, pp. 431-446, 2005.

[17] T. Floquet, C. Edwards, and S. K. Spurgeon, "On sliding mode observers for systems with unknown inputs," International Journal of Adaptive Control and Signal Processing, vol. 21, pp. 63-65, 2007.

[18] P. A. Ionnou and P. V. Kokotovic, "Instability analysis and improvement adaptive control of robustness of adaptive control," Automatica, vol. 20, no. 5, pp. 583-594, 1984.

[19] M. Corless and J. Tu, "State and input estimation for a class of uncertain systems," Automatica, vol. 34, pp. 757-764, 1998.

[20] J. H. Ahrens and H. K. Khalil, "High-gain observers in the presence of measurement noise: A switched-gain approach," Automatica, vol. 09, pp. 936-943, 2015.

[21] C. Tréangle, M. Farza, and M. M'Saad, "A simple filtered high gain observer for a class of uncertain nonlinear systems," in 18th international conference on Sciences and Techniques of Automatic control and computer engineering, (Monastir, Tunisia), pp. 396401, 2017.

[22] V. V. Assche, T. Ahmed-Ali, C. A. B. Hann, and F. Lamnabhi-Lagarrigue, "High gain observer design for nonlinear systems with time varying delayed measurements," in Proc. 18th IFAC world congress, (Milano, Italia), pp. 692-696, 2011.

[23] M. Farza, O. Hernandez-Gonzalez, B. T. T. Ménard, M. M'Saad, and C. M. AstorgaZaragoza, "Cascade observer design for a class of uncertain nonlinear systems with delayed outputs," Automatica, vol. 89, pp. 125-134, 2018. 